Case Report:

\title{
A Rare Case of Sacrococcygeal Teratoma With Medullar Invasion
}

Najoua Aballa ${ }^{1}$ (D) Houssine Ghannane ${ }^{1}$ (D) Mohammed Oulad Saiad $^{1^{*}}$ (D)

1. Department of Pediatric Surgery, School of Medicine, Mother and Child Hospital, Mohammed VI Teaching Hospital, University Cadi Ayyad, Marrakesh, Morocco

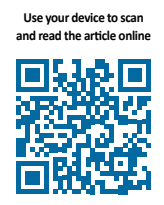

Citation: Aballa N, Ghannane H, Oulad Saiad M. A Rare Case of Sacrococcygeal Teratoma With Medullar Invasion. Iran J Neurosurg. 2020; 6(4):229-232. http://dx.doi.org/10.32598/irjns.6.4.8

dol: $:$ http://dx.doi.org/10.32598/irjns.6.4.8

\section{(c) (i) (\$)}

Article info:

Received: 19 Jun 2020

Accepted: 16 Aug 2020

Available Online: 01 Oct 2020

Keywords:

Sacrococcygeal teratoma,

Medular invasion, Neurological

deficit, Alpha-fetoprotein,

Malignancy

\section{ABSTRACT}

Background and Importance: Sacrococcygeal Teratoma (SCT) with medular invasion is rare Case Presentation: We report a case of an 11-month male infant, with no prenatal history of any abnormality, presenting since birth, a mass in the buttock extended to retrorectum associated with a right side hypotonic limb and monoplegia. Medullar and abdominal pelvic Magnetic Resonance Imaging (MRI) showed a sacrococcygeal tissue mass and intradural lumbosacral invasion with a high level of alpha-fetoprotein. Surgical excision was done successfully with an uneventful follow-up. Conclusion: Despite of the intradural invasion, those SCT are in major cases mature and present low risk of malignancy or recurrence.

\section{* Corresponding Author:}

Mohamed Oulad Saiad, MD.

Address: Department of Pediatric Surgery, School of Medicine, Mother and Child Hospital, Mohammed VI Teaching Hospital, University Cadi Ayyad, Marrakesh, Morocco

Tel: +212 (67) 3083983

E-mail: mouladsaiad@gmail.com 


\section{Highlights}

- Sacrococcygeal Teratoma (SCT) is the most common type of teratoma, developed from the coccyx.

- Sacrococcygeal teratoma with medullar extension is rare.

- It should be managed adopting a multidisciplinary approach with joint cooperation of pediatric surgeon, neurosurgeon, and radiologist to minimize the risk of permanent neurological deficits and recurrence.

\section{Plain Language Summary}

The most common type of teratoma is Sacrococcygeal Teratoma (SCT) which stems from the coccyx. Mature sacrococcygeal teratoma is more common in neonates and infants. Surgical excision is the recommended treatment. The extension of the tumor to the medullar canal is rare. A few cases have been reported so far. Physical examination in these patients may show neurological deficit as paraplegia or can be normal.

\section{Background and Importance}

acrococcygeal Teratoma (SCT) with me-

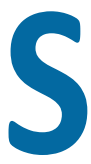
dular invasion is rare [1] and is the most common type of teratoma, developed from the coccyx. It contains the 3 embryological layers and can be mature benign or immature malign. Likely mature sacrococcygeal teratoma is more frequent in neonates and infants, it comprises $60 \%$ of teratomas [2]. Therefore, surgical excision of the tumor is the main treatment of this tumor. The extension of the tumor to the medullar canal is rare [2, 3]. Only a few cases have been reported in the literature and physical examination in those cases can find a neurological deficit as paraplegia or can be normal. We performed a review of the literature to discuss this issue.

\section{Case Presentation}

Our case was an 11-month male infant, second parity of consanguineous couple, who was born at 35 weeks of pregnancy by C-section following an uneventful pregnancy. No abnormality was noted on prenatal obstetrical ultrasonography. Physical examination found a regular mass in the right buttock that displaced the anus interiorly; without lesion of the skin covering the mass. He had a hypotonic right monoplegia of the right inferior limb and also presented the difficulty of defecation but normal urination. Cerebrospinal and pelvic MRI revealed a tissue mass of the right buttock with the endopelvic extension on the right ischioanal fossa. This mass contains cystic zones and calcifications and progresses on the intradular area until the lumbar level measuring
$92 \times 40 \times 52 \mathrm{~mm}$. It causes osteolysis of sacral and coccygeal parts and enlarges the spinal canal (Figure 1).

Surgery was made by a vertical median lumbosacral approach, dissection of both endo- and exopelvic components. The intradular part of the tumor extended to the $3^{\text {rd }}$ lumbar vertebra was also dissected after a dural sac opening by a posterior approach (Figure 2).

Total excision of the tumor was done by taking the coccyx. Histopathology showed a mature tumor without any sign of malignancy. Seven months after surgery, the patient still presents deficit in his right lower limb, with no recurrence at the control MRI.

\section{Discussion}

SCT arises from embryologically multipotent cells which reside within the coccyx [3]. SCT can be mature (benign) or immature (malign). Sacrococcygeal teratoma is the most common teratoma in infants, more frequent than intradural teratoma. Sacrococcygeal teratoma with medullar invasion is on the other hand rare. Few cases have been described. Altman et al. reported no occurrence in 405 cases, Gross et al. reported 2 of 40 cases without describing details, Donnellan and Swensen reported 3 of 54 cases but described only dural sac opening [4]. The tumor is in major cases attached to the filum terminale. In our case, the tumor was also attached to the filum terminale and caused osteolysis of sacral and coccygeal vertebras. The intradural invasion can be explained by an aggressive immature teratoma or growth of the tumor in a less resistant area. SCT complicated by spinal metastases has the same presentation of spinal invasion of SCT. Imaging evaluates the exo- 


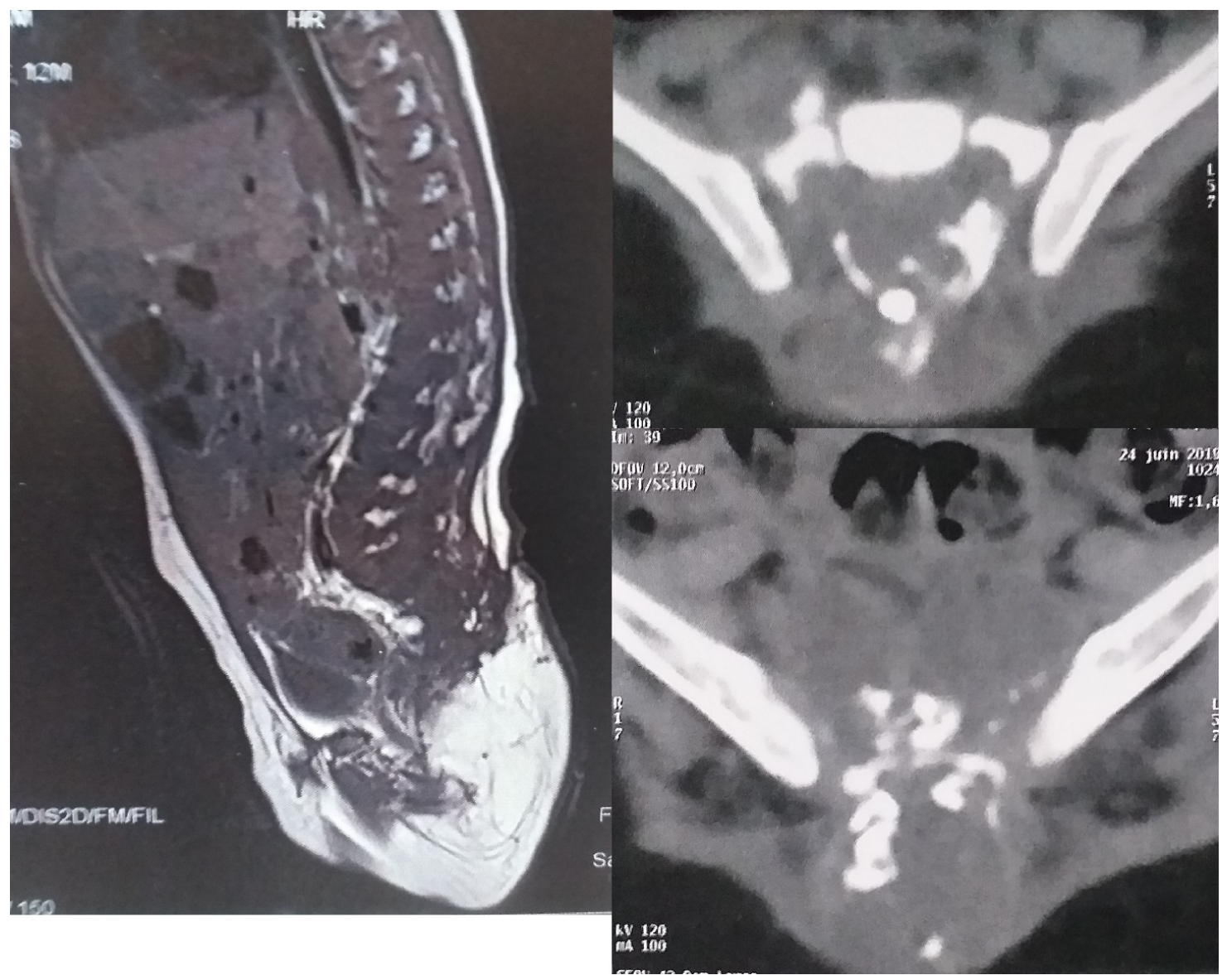

No

Figure 1. Sagittal and coronal view of pre-operative magnetic resonance imaging showing osteolysis of the sacral and coccygeal bone

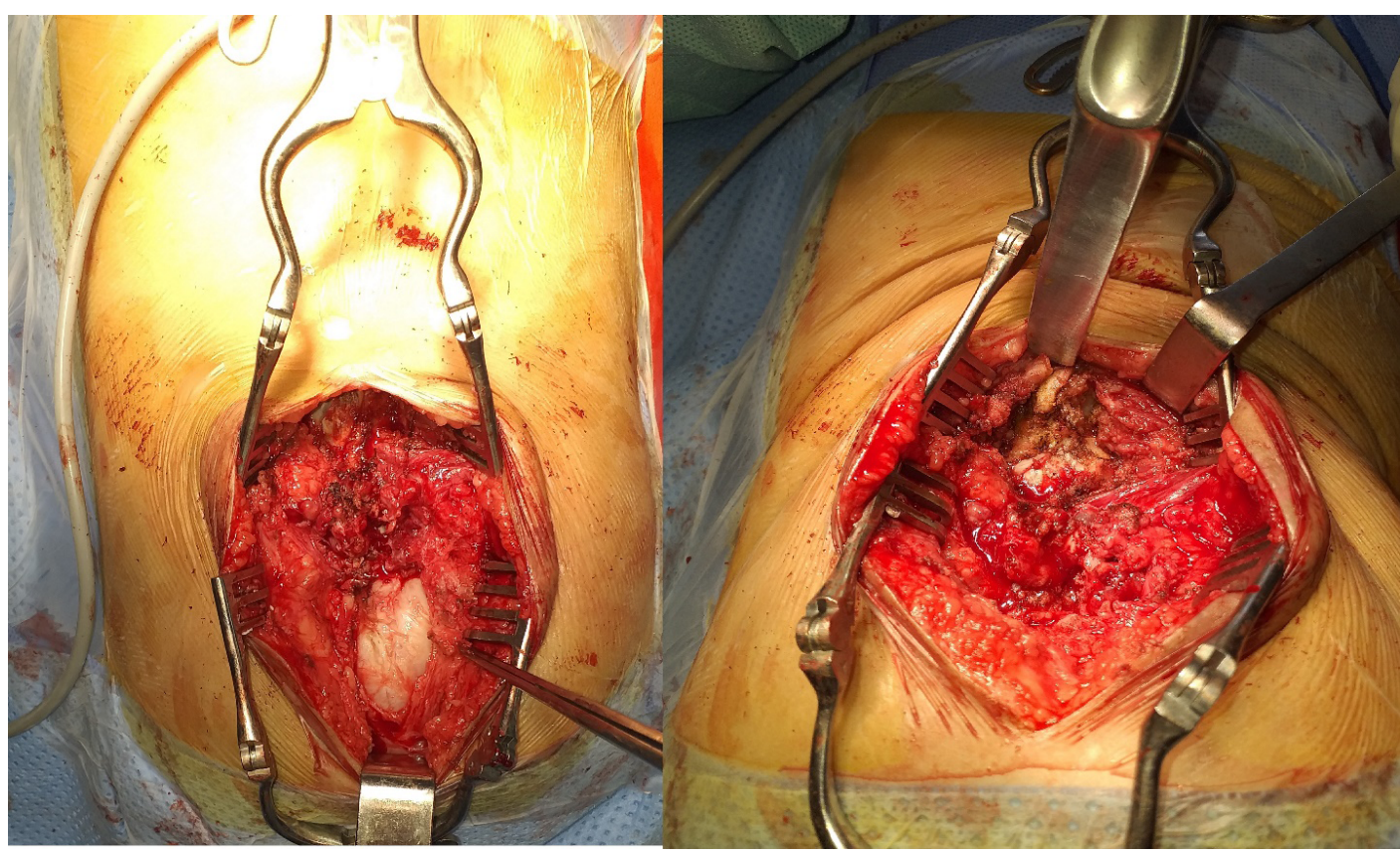

Figure 2. Intra-operative view of sacroccygeal teratoma with intramedular invasion 
pelvic and intra-abdominal parts of the tumor and also shows the presacral component and reveals if the deficit is due to intradural extension or spinal metastases. MRI is the best way to explore SCT with neurological deficit, and aids to establish the management of surgery. Ultrasonography can explore not only the abdominal part of SCT but also the involvement of the spinal canal.

Prenatal diagnosis of SCT is associated with a high risk of mortality due to heart failure caused by arteriovenous shunt causing vascular steal. After birth, the prognosis is good after total tumor excision. Severe neurological deficit can be present from birth or appear post-operatively, Ribeiro et al. [5] presented a case of intradural and extradural extension of an SCT, 2 years after complete excision, the patient developed a neurogenic bladder with fecal retention. Kunisaki et al. [3], presented a case of a neonate with SCT complicated by an intradural invasion who had since birth a paraplegia that became permanent after surgery and developed also a neurogenic bladder due to prolonged compression of the conus medullaris. Early diagnosis of this entity can provide the risk of permanent neurological sequelae. Intradullar invasion is not especially a risk factor of malignancy or recurrence, but more studies with a long-term follow-up are required to establish the prognosis of those cases.

\section{Conclusion}

Sacrococcygeal teratoma with medullar extension is rare, and must be managed with a multidisciplinary approach, associating pediatric surgeon, neurosurgeon, and radiologist to minimize the risk of permanent neurological deficit and recurrence. The invasion of the spinal canal does not increase the risk of malignancy.

\section{Ethical Considerations}

Compliance with ethical guidelines

Informed consent was obtained from the patient's parents.

Funding

This research did not receive any grant from funding agencies in the public, commercial, or non-profit sectors.

\section{Authors contributions}

All authors equally contributed to preparing this article.

\section{Conflict of interest}

The authors declared no conflict of interest.

\section{References:}

[1] Shahjouei S, Hanaei S, Nejat F, Monajemzadeh M, Khashab ME. Sacrococcygeal teratoma with intradural extension: Case report. Journal of Neurosurgery. Pediatrics. 2015; 15(4):380-3. [DOI:10.3171/2014.10.PEDS1445] [PMID]

[2] Jelin E, Jelin AC, Lee H. Sacrococcygeal teratoma with spinal canal invasion prenatally diagnosed. Journal of Pediatric Surgery. 2009; 44(4):E9-11. [DOI:10.1016/j.jpedsurg.2008.12.027] [PMID]

[3] Kunisaki SM, Maher CO, Powelson I, Gemmete JJ, Hirsch RB, Mychaliska GB. Benign sacrococcygeal teratoma with spinal canal invasion and paraplegia. Journal of Pediatric Surgery. 2011; 46(9):e1-4. [DOI:10.1016/j.jpedsurg.2011.05.013] [PMID]

[4] Powell RW, Weber ED, Manci EA. Intradural extension of a sacrococcygeal teratoma. Journalof Pediatric Surgery. 1993; 28(6):770-2. [DOI:10.1016/0022-3468(93)90322-C]

[5] Ribeiro PR, Guys JM, Lena G. Sacrococcygeal teratoma with an intradural and extramedullary extension in a neonate: Case report. Neurosurgery. 1999; 44(2):398-400. [DOI:10.1097/00006123-199902000-00094] [PMID] 